\title{
Processos não Hidrolíticos Sol-Gel: Conceitos e Aplicações em Catálise Heterogênea
}

\author{
César A. S. Henriques, Lucas D. Dias, \\ Mário J. F. Calvete \& Gilberto L. B. de Aquino
}

O desenvolvimento de novos materiais é um tópico de relevância no âmbito das ciências dos materiais, em razão da importante função desempenhada em diversas aplicações na sociedade moderna. No entanto, existe grande preocupação e necessidade no desenvolvimento de processos eficientes e alternativos aos processos sol-gel, sendo este o mais utilizado na síntese de catalisadores heterogêneos. Desse modo, o objetivo deste trabalho é proporcionar um estudo geral sobre os princípios da via alternativa não hidrolítica sol-gel (NHSG), suas potencialidades e vantagens, tais como o controle da estrutura e composição, mostrando ser um método mais eficiente que o processo sol-gel convencional.

Palavras-chaves: não hidroliticos sol-gel; materiais porosos; catálise heterogênea.

The development of new materials is a topic of relevance on the ambit of science materials, because of the important role played in several applications in modern society. However, there is a major concern and necessitate in development of process efficient and alternatives to sol-gel process, it is the most used on the synthesis of heterogeneous catalysis. Thus the objective this work is afford a general study about the principles of the via alternative non-hydrolytic sol-gel (NHSG), its potential and advantages, such as the control of structure and composition, showing that must efficient method than the conventional sol-gel process.

Keywords: Non-hydrolytic sol-gel; porous materials; heterogeneous catalysis. 


\section{Introdução}

O desenvolvimento de materiais é, atualmente, um tópico de extrema relevância no campo das ciências dos materiais, devido ao papel que estes desempenham na sociedade moderna. Contudo, materiais cerâmicos, obtidos por processos sol-gel, há muito que são utilizados no nosso quotidiano.

O método de produção de cerâmica - método da cerâmica - utiliza condições reacionais severas que apenas conduzem a fases termodinamicamente estáveis, impedindo a formação de sólidos metaestáveis. Correntemente, a química orgânica oferece-nos vias sintéticas, altamente sofisticadas, capazes de quebrar ligações químicas de forma controlada, fazendo do método da cerâmica uma abordagem bastante grosseira. Assim, não é surpreendente que novas abordagens de síntese de materiais porosos tenham surgido, possibilitando a obtenção de sólidos com morfologias cristalinas bem definidas, uniformes e com uma maior homogeneidade. ${ }^{1}$ Porém, a influência de diversos parâmetros torna a formação da rede inorgânica um processo complexo, sendo, portanto, necessária uma compreensão detalhada dos padrões que influenciam a taxa e o curso da formação dos géis para moldar as propriedades finais do material.

Está bem estabelecido que os fatores que influenciam o delineamento dos materiais são o tipo de percursor e de catalisador, a razão entre os reagentes, o tipo de solvente, a temperatura, o $\mathrm{pH}$ e as concentrações dos componentes em misturas de precursores ${ }^{2}$. O estudo pormenorizado destes fatores, resultado da forte dedicação que a comunidade científica tem dado a esta área, permitiu uma grande evolução no processo sol-gel que, presentemente, pode ser dividido em duas principais rotas: a rota hidrolítica sol-gel e a rota não hidrolítica sol-gel. Ambas têm como finalidade a síntese de materiais através da conversão de uma solução coloidal num sistema de rede tridimensional (gel) diferenciando-se, contudo, pela estratégia adotada.

A rota hidrolítica sol-gel ou processo sol-gel aquoso, como o próprio nome indica, utiliza água não só como solvente mas também como ligante. Em geral, são usados sais metálicos inorgânicos (tais como cloretos, nitratos, sulfatos) ou alcóxidos de metal como precursores. Estes últimos são os mais amplamente utilizados, uma vez que reagem facilmente com água, numa etapa denominada por hidrólise, formando uma sol por polimerização - via condensação - cuja continuação do processo leva à formação de um gel. A rapidez do processo, diretamente relacionada com o passo da hidrólise, constitui umas das principais limitações desta abordagem, pois o controle estrutural, da morfologia, da composição e da homogeneidade do material é restrito. Para colmatar este problema, podem utilizar-se estratégias alternativas, como a realização da pré-hidrólise ácida dos precursores menos reativos ${ }^{3}$ ou a modificação química dos mais reativos ${ }^{4}$. Outro problema do processo sol-gel aquoso é a precipitação do gel diretamente no meio reacional levando à formação de um material, geralmente, amorfo. Este pode, posteriormente, ser seco por evaporação (recorrendo-se, por vezes, à temperatura) o que muitas vezes conduz ao colapso dos poros da rede, não permitindo, assim, o controle sobre o tamanho e forma do cristal. Neste contexto, e de forma a contornar esta dificuldade, Pajonk ${ }^{5}$ utilizou dispositivos de secagem supercríticos. $\mathrm{Rao}^{6}$, por sua vez, procedeu a uma troca de solvente, bem como, à derivatização da superfície hidroxilada por sililação, antes do passo da evaporação. Recentemente, outros autores ${ }^{7,8}$, utilizaram agentes de molde para preparar materiais mesoporosos ordenados e combinaram esta estratégia com o processamento aerossol para permitir a texturização em multi-escala 9 .

Apesar de todos os esforços realizados para contornar os problemas do processo aquoso sol-gel, a forte sensibilidade a pequenas alterações na síntese e a ocorrência simultânea de reações de hidrólise e de condensação torna o controle do processamento de óxidos de metal em meio aquoso muito difícil.

Assim, nas duas últimas décadas, tem sido realizado um enorme esforço de forma a encontrar alternativas à rota sol-gel aquosa. Foi, neste âmbito, que Vioux e os seus colaboradores sugeriram, pela primeira vez, o termo "sol-gel não hidrolítico" (NHSG, do inglês "Nonhydrolytic sol-gel"). 10,11,12. Esta nova aproximação tem sido utilizada na preparação de óxidos, ${ }^{13,14}$ híbridos orgânicosinorgânicos, ${ }^{15,16}$ nanopartículas de óxidos, ${ }^{17,18}$ filmes por deposição de camada atómica ${ }^{19,20}$ enanoestruturas..$^{21,22}$ Neste trabalho, serão abordados os princípios da rota NHSG, com o intuito de mostrar quais as suas potencialidades e vantagens relativamente ao processo sol-gel convencional. Por fim, será apresentada a aplicação do processo NHSG na preparação de catalisadores heterogêneos. 


\section{Rota de condensação não hidrolítica}

\section{REAÇÕES CONDENSAÇÃO NÃO HIDROLÍTICA}

Esta rota pode ser definida como a conversão de um precursor molecular, dissolvido num solvente orgânico, num sólido inorgânico sem utilização de água como doador de oxigênio. Para além desta diferença fundamental, relativamente ao processo solgel aquoso, a lista de potenciais precursores também é consideravelmente diferente. Para esta rota, a lista é maior e inclui, para além de sais metálicos inorgânicos e alcóxidos de metais, acetatos e acetilacetonatos de metais. Quanto ao doador de oxigênio, necessário para a formação do óxido de metal, este pode ser fornecido pelo solvente orgânico utilizado (éteres, álcoois, cetonas ou aldeídos) ou pelo componente orgânico do precursor (alcóxidos ou acetilacetonatos). Assim, a formação da ligação metal-oxigénio-metal, unidade estrutural destes materiais, pode ser efetuada através das seguintes vias.

\section{Eliminação de haletos de alquilo}

As primeiras reações a serem estudadas foram as condensações não hidrolíticas envolvendo eliminação de haletos de alquilo, através da reação entre alcóxidos e haletos metálicos (eq. 1), devido à ampla disponibilidade e baixo custo dos haletos metálicos.

$$
\mathrm{M}-\mathrm{X}+\mathrm{M}-\mathrm{OR} \longrightarrow \mathrm{M}-\mathrm{O}-\mathrm{M}+\mathrm{R}-\mathrm{X}
$$

No caso de metais e metais de transição, esta reação ocorre a temperaturas moderadas $\left(80-150^{\circ} \mathrm{C}\right)$, com grupos alquílicos primários ou secundários, embora seja necessária maior energia de ativação que no processo hidrolítico. A velocidade de reação é ainda mais baixa, no caso do silício, que necessita de um ácido de Lewis, tal como o $\mathrm{FeCl}_{3}$ ou $\mathrm{AlCl}_{3}$, para catalisar a condensação. ${ }^{23}$

Alternativamente, as espécies de alcóxido de metal podem também ser produzidas in situ através da reação de haletos metálicos com éteres (eq. 2) ou com álcoois (eq. 3), o que é de particular interesse se o alcóxido for caro ou não estiver disponível comercialmente.

$$
\begin{aligned}
& \mathrm{M}-\mathrm{X}+\mathrm{ROR} \longrightarrow \mathrm{M}-\mathrm{OR}+\mathrm{R}-\mathrm{X} \\
& \mathrm{M}-\mathrm{X}+\mathrm{ROH} \longrightarrow \mathrm{M}-\mathrm{OR}+\mathrm{H}-\mathrm{X}
\end{aligned}
$$

As espécies de alcóxido de metal reagem seguidamente da maneira apresentada na eq. 1 para formar as pontes oxo. As vias alcóxido e a via na qual a espécie alcóxido é , por reação de haletos metálicos com éteres, têm se mostrado bastante importantes no controle da homogeneidade e textura na síntese de mistura de óxidos. ${ }^{24,25,26,27,28}$.

Por sua vez, na rota em que o álcool é utilizado como precursor, as reações envolvidas dependem do tipo de álcoois. Com álcoois primários e secundários, formam-se grupos alcóxidos (eq. 3) que, num segundo passo, reagem com haletos metálicos de acordo com a eq. 1. Os álcoois terciários e benzílicos podem, também, levar à formação de grupos hidroxilos (eq. 4), que reagem depois com os haletos de metal de acordo com a equação ${ }^{2.5}$.

$$
\begin{aligned}
& \mathrm{M}-\mathrm{X}+\mathrm{R}-\mathrm{OH} \longrightarrow \mathrm{M}-\mathrm{OH}+\mathrm{R}-\mathrm{X} \\
& \mathrm{M}-\mathrm{X}+\mathrm{M}-\mathrm{OH} \longrightarrow \mathrm{M}-\mathrm{O}-\mathrm{M}+\mathrm{H}-\mathrm{X}
\end{aligned}
$$

Note-se que a reação do haleto de metal com álcoois (eq. 3) ou com hidróxido metálico (eq. 5) liberta ácido que pode catalisar reações secundárias, tais como a eliminação de grupos éter, que conduz a vias complexas (como mostra o tópico seguinte).

\section{Eliminação de éteres}

A condensação entre dois alcóxidos de metal resulta na eliminação de éteres e na formação do respetivo óxido de metal (eq. 6). Esta reação requer uma temperatura relativamente elevada, razão pela qual se tem verificado uma decomposição térmica em vez do processamento sol-gel. ${ }^{29}$

$$
\mathrm{M}-\mathrm{OR}+\mathrm{M}-\mathrm{OR} \longrightarrow \mathrm{M}-\mathrm{O}-\mathrm{M}+\mathrm{R}-\mathrm{O}-\mathrm{R}
$$


$\mathrm{O}$ interesse nos aspetos mecanísticos da transformação de alcóxido de metal em oxo-alcóxido, como intermediários, levou à descoberta deste processo. As reações de eliminação de éteres são caraterísticas de metais de transição como $\mathrm{Mo}^{\mathrm{VI}}, \mathrm{W}^{\mathrm{VI}}$ e $\mathrm{Nb}^{\mathrm{V}}$, nas quais o material inorgânico é diretamente produzido na presença de precursores alcóxidos básicos. Na literatura, foi possível observar também a formação de espécies oxo e eliminação de éteres através da reação entre alcóxidos metálicos e aldeídos ou cetonas. ${ }^{30,31}$

\section{Eliminação de ésteres}

A condensação direta entre carboxilatos de metal e alcóxidos de metal, em solventes apolares e apróticos, permite a obtenção de óxidos de metal através da rota não hidrolítica (eq. 7).

\section{$\mathrm{M}-\mathrm{OCOR}^{2}+\mathrm{M}-\mathrm{OR}^{1} \longrightarrow \mathrm{M}-\mathrm{O}-\mathrm{M}+\mathrm{R}^{2} \mathrm{COOR}^{1} \quad$ (Eq. 7)}

O primeiro passo da reação entre o alcóxido e o ácido carboxílico resulta na libertação de um álcool, através da troca de grupos carboxilatos e alcoxi no metal (eq. 8). ${ }^{32}$

$\mathrm{M}\left(\mathrm{OR}^{1}\right)_{\mathrm{n}}+\mathrm{R}^{2} \mathrm{COOH} \longrightarrow \mathrm{M}\left(\mathrm{OR}^{1}\right)_{\mathrm{n}-1}\left(\mathrm{OCOR}^{2}\right)+\mathrm{R}^{1} \mathrm{OH}$

Apesar da condensação aprótica direta (como descrito na equação 2.7) não poder ser descartada, a hidroxilação não hidrolítica de derivados carboxilato com álcool formado na reação (eq. 9) ${ }^{42}$ é mais provável de ocorrer, assim como, a esterificação do ácido carboxílico (eq. 10), com a formação in situ de água, ${ }^{33}$ o que faz com que o processo possa não ser estritamente não hidrolítico, pelo menos em solução.

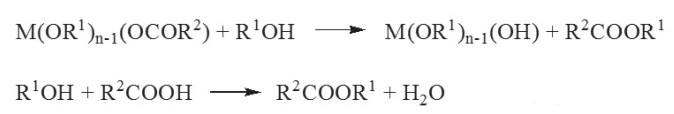

Contudo, com base em considerações cinéticas, vários autores, defendem que a formação de óxidos de metal por esta via envolve principalmente acetoxilação (eq. 8), seguida de condensação aprótica (eq. 7).
A reação de alcóxidos com ácidos carboxílicos é particularmente interessante com precursores de alto peso molecular, em que a solubilidade é limitada, ou em sínteses em meios alternativos como solventes perfluorados, líquidos iónicos ${ }^{37,38} \mathrm{e} \mathrm{CO}_{2}$ supercrítico $^{39}$ para realizar a síntese sol-gel.

Alternativamente, pode fazer-se uso da reação de alcóxidos de metal com anidridos (eq. 11) que conduz à formação in situ de grupos carboxilato, que podem,então, condensar com alcóxidos (eq. 7), proporcionando uma via inequívoca de sol-gel não hidrolítica.

$\mathrm{M}\left(\mathrm{OR}^{1}\right)_{\mathrm{n}}+\left(\mathrm{R}^{2} \mathrm{CO}\right)_{2} \mathrm{O} \longrightarrow \mathrm{M}\left(\mathrm{OR}^{1}\right)_{\mathrm{n}-1}\left(\mathrm{OCOR}^{2}\right)+\mathrm{R}^{2} \mathrm{COOR}^{1}$

Esta aproximação foi usada, com sucesso, na preparação de óxido de titânio ${ }^{40}$ (utilizando o $\mathrm{TiCl}_{4}$ como catalisador) e mistura de óxidos de titânio e sílica. ${ }^{41}$

\section{Eliminação de amidas}

Outra reação de hidroxilação não hidrolítica é providenciada pela eliminação de amida de precursores carboxilados (eq. 12).

$\mathrm{M}\left(\mathrm{OR}^{1}\right)_{\mathrm{n}-1}\left(\mathrm{OCOR}^{2}\right)+\mathrm{R}^{3} \mathrm{NH}_{2} \longrightarrow \mathrm{M}\left(\mathrm{OR}^{1}\right)_{\mathrm{n}-1}(\mathrm{OH})+\mathrm{R}^{2} \mathrm{CONHR}^{3}$

\section{Condensação Aldólica}

Cetonas e aldeídos podem ser usados como doadores de oxigênio em processos não hidrolítcos sol-gel embora possam gerar água in situ aquando a formação de compostos carbonilo $\alpha, \beta$-insaturados, esquema 1 . No entanto, tal como na esterificação e na desidratação de álcoois, a eliminação da água pode ser considerada apenas formal, na presença de uma grande quantidade de metais.

2
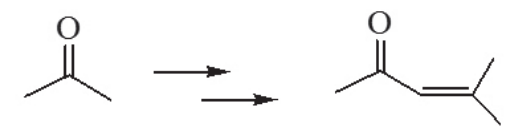

$+\mathrm{H}_{2} \mathrm{O}$

Esquema 1: Reação geral na formação de compostos carbonilo $\alpha, \beta$ insaturados, através de cetona.

Apesar da limitação da utilização de cetonas e aldeídos como precursores para a transformação solgel, vários autores ${ }^{44,45,46}$ têm preparado óxidos metálicos utilizando esta estratégia, uma vez que acetilacetonatos de metais estão, para alguns metais, mais disponíveis que os alcóxidos. 


\section{Eliminação de álcoois envolvendo a formação de ligações $\boldsymbol{C}$-C}

Esta rota centra-se na reação de álcoois primários, tais como álcool benzílico e o álcool $p$-metoxibenzílico, com alcóxidos de metal na presença de espécies básicas, cuja reação é diferente da anteriormente apresentada que resulta na eliminação de éteres. A química mecanística deste processo é análoga à proposta por Guerbet, ${ }^{47} \mathrm{em}$ que o alcóxido de metal alcalino reage com o álcool benzílico, por meio de um mecanismo de acoplamento $\mathrm{C}-\mathrm{C}$, que leva à formação de um novo álcool, de maior peso molecular, e do respetivo óxido de metal, como mostra o esquema 2.
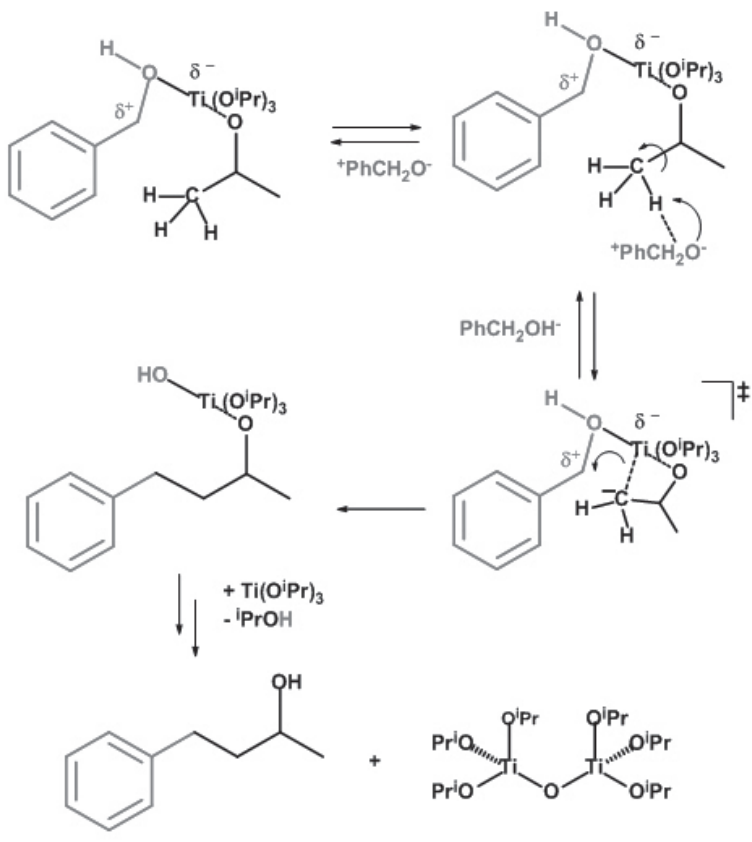

Esquema 2: Proposta mecanística para a eliminação do álcool envolvendo a formação de ligações C-C.

Comparativamente com a reação de alcóxidos metálicos com álcoois, apresentada na secção 2.1.2, que usualmente prossegue através da eliminação do éter, a maior diferença do ponto de vista experimental é a dissolução de bário metálico no álcool antes da adição do álcoxido de metal. No entanto, o mecanismo de reação é completamente alterado e apenas quantidades vestigiais de éteres foram encontradas no final da reação. ${ }^{48}$

\section{Decomposição térmica}

Os caminhos e mecanismos discutidos anteriormente dependem de reações entre a espécie precursora e o solvente ou entre duas espécies precursoras a temperaturas abaixo dos $350^{\circ} \mathrm{C}$. Altas temperaturas conduzem ao aumento das vias reacionais, bem como, da sua complexidade e podem resultar, por vezes, na decomposição térmica dos precursores.

Por exemplo, a decomposição térmica dos precursores de alcóxido envolve geralmente a eliminação de alcenos e formação de grupos hidroxilo que, de seguida, podem condensar com outros alcóxidos e formar pontes oxo com a eliminação do álcool. Por sua vez, os grupos hidroxilos ,formados através da decomposição térmica, podem também condensar entre si, levando à formação de água. Contudo, como a condensação entre grupos $\mathrm{M}-\mathrm{OH}$ é cineticamente impedida, estas reações de decomposição podem ser consideradas não-hidrolíticas.

Assim, diversas rotas não hidrolíticas baseadas na decomposição térmica de vários precursores, a relativamente altas temperaturas, têm sido empregadas na preparação de nanopartículas, ${ }^{49,50,51}$, mistura de óxidos ${ }^{52,53}$ e ligação de espécies catalíticas à superfície de suportes de sílica. ${ }^{54,55}$

\section{CONTROLE DA COMPOSIÇÃO E HOMOGENEIDADE}

A condensação não hidrolítica tem por base uma química que permite um maior controle sobre a cinética das reações comparativamente com a rota hidrolítica, uma vez que apresentam maior energia de ativação. Por esta razão, não é necessária a utilização de modificadores de reatividade que normalmente requer procedimentos elaborados. Tendo em conta estes fatos, é possível, por este método, a preparação de matérias de mistura de óxidos ou híbridos orgânico-inorgânico bastante homogêneos, que dificilmente seriam obtidos pela rota hidrolítica.

\section{Óxidos e mistura de óxidos de metais}

A preparação de materiais porosos com elevada homogeneidade e relações definidas de mistura de óxidos de metais ficou facilitada com a utilização de meios não hidrolíticos. A utilização de rotas não hidrolíticas permite a obtenção de elevada homogeneidade molecular na 
mistura de óxidos de metais devido à formação de fases metaestáveis à baixa temperatura. Exemplo disso foi a preparação de vários sistemas binários $\left(\mathrm{Si}-\mathrm{Ti},{ }^{34,56,57} \mathrm{Si}\right.$ $\mathrm{Zr},{ }^{67} \mathrm{Si}-\mathrm{Al},{ }^{58,59} \mathrm{Sn}-\mathrm{Al},{ }^{60} \mathrm{Ti}-\mathrm{Al},{ }^{36,61,62} \mathrm{Ti}-\mathrm{Zr},{ }^{35,63} \mathrm{Zr}-\mathrm{Mo},{ }^{37}$ Ga-Mo, ${ }^{38} \mathrm{~V}-\mathrm{Nb},{ }^{64} \mathrm{Fe}^{-\mathrm{Ti}^{65}}$ e Ti-V ${ }^{66,67}$ ), com um excelente controle sobre a estequiometria e homogeneidade dos óxidos mistos, utilizando a via da eliminação do halogeneto de alquilo. O mesmo método foi também aplicado a certos sistemas ternários, como V-Si-Nb, ${ }^{74} \mathrm{Co}-\mathrm{Al}-\mathrm{Si},{ }^{68}$ e até mesmo quartenários, $\mathrm{Na}_{3} \mathrm{Zr}_{2} \mathrm{Si}_{2} \mathrm{PO}_{12}{ }^{69} \mathrm{Na}$ preparação do sistema binário, $\mathrm{Al}-\mathrm{Ti}(\mathrm{Al} / \mathrm{Ti}=2)$ foi obtida, por cristalização direta, uma fase metaestável pseudobrookite $\beta$ - $\mathrm{TiAl}_{2} \mathrm{O}_{5}$, em vez das fases estáveis de rutilo e corindo. Este comportamento demonstra que, partindo de um gel homogêneo, a cristalização para um óxido misto metaestável, requer uma menor distância de difusão do que para a separação de fases para as fases estáveis sendo, portanto, significativamente mais rápida. Pela via do éter (eq. 2.6), também foi possível a síntese de outras fases metaestáveis, como do $\mathrm{ZrW}_{2} \mathrm{O}_{8}{ }^{37}$ e $\mathrm{Ga}_{2} \mathrm{Mo}_{3} \mathrm{O}_{12}{ }^{38}$

A elevada homogeneidade permite aumentar a atividade catalítica dos materiais, como constatou Vioux e os seus colaboradores ${ }^{70,71,72}$ aquando a preparação de óxidos mistos de Si-Ti para aplicação em oxidações. A rota não hidrolítica impede a formação de $\mathrm{TiO}_{2}$ e assim os locais de titânio na rede estão mais disponíveis para catalisar oxidações.

A calcinação a que os xerogéis são normalmente submetidos pode ser vantajosa para as suas potenciais aplicações uma vez que esta pode alterar a composição e homogeneidade dos materiais. Exemplo disso é calcinação a $500^{\circ} \mathrm{C}$ a que os catalisadores de V-Ti são submetidos, que faz com que as espécies de Vanádio migrem para a superfície, figura 1 , devido à sua baixa temperatura Tammann (temperatura à qual os átomos apresentam mobilidade). ${ }^{77}$ Esta abordagem permitiu resolver algumas lacunas das abordagens clássicas em que catalisadores eram preparados através da impregnação de um precursor de titânio em sílica, seguida da deposição de um precursor de vanádio. Esta via apresenta algumas limitações, pois as espécies impregnadas possuem tendência a aglomerarem sobre a superfície de apoio. ${ }^{73} \mathrm{O}$ aumento da atividade catalítica em reações de oxidação também foi observado em materiais inorgânicos de Si-Ti após calcinação, devido à baixa densidade de silanóis. ${ }^{81}$

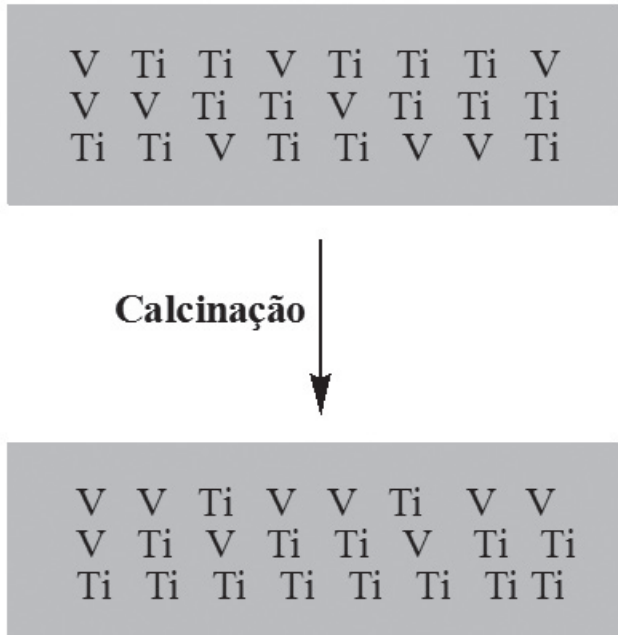

Figura 1: Migração das espécies de vanádio para a superfície após calcinação a $500^{\circ} \mathrm{C}$.

\section{Materiais híbridos orgânico-inorgânicos}

Embora, para este tipo de materiais, as condições de reação sejam menos suaves do que no processo hidrolítico sol-gel, as vias não hidrolíticas podem ser utilizadas para a encapsulação ou imobilização covalentemente de espécies orgânicas ou organometálicas em matrizes de óxido. Nos últimos anos, têm sido preparados diversos materiais híbridos orgânico-inorgânicos, de forma a contornar alguns problemas, tais como a fraca solubilidade de componentes orgânicos em água e a separação de fases entre os componentes orgânicos e inorgânicos. As reações de condensação não hidrolíticas são particularmente úteis para preparar materiais híbridos orgânico-inorgânicos transparentes cujas propriedades podem satisfazer aos requisitos para aplicações em fotónica, tais como um baixo teor de hidroxilos e um elevado grau de homogeneidade.

Para além da aplicação na área da fotónica, diversos catalisadores híbridos orgânico-inorgânicos têm também sido preparados por incorporação de espécis orgânicos em materiais inorgânicos. Uma estratégia que tem sido adoptada é a encapsulação de complexos metálicos, como metaloporfirinas ou catalisador de Jacobsen - figura 2 - em matrizes de alumina, no poro do material. ${ }^{74,75,76}$ 


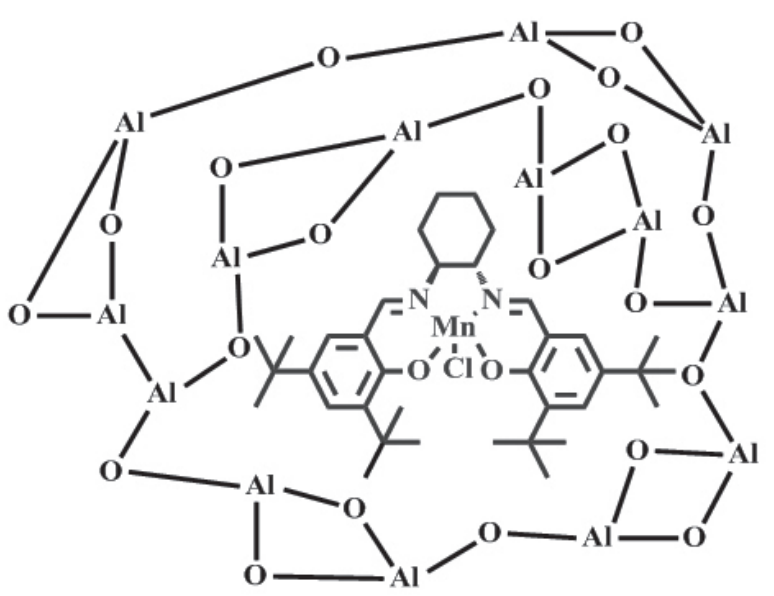

Figura 2: Representação esquemática do catalisador de Jacobsen encapsulado numa matriz de alumina

A ligação covalente de organossilanos $\mathrm{RSiX}_{3}$ $(\mathrm{X}=\mathrm{Cl}, \mathrm{OR}, \mathrm{H})$ à superfície hidroxilada não pode ser considerada não hidrolítica, na verdade, a diminuição da densidade das monocamadas com a água, presente no meio reacional, indica que a hidrólise dos grupos $\mathrm{Si}-\mathrm{X}$ pela água adsorvida está, provavelmente, envolvida no primeiro passo. ${ }^{77,78,79}$ Por outro lado, a modificação do óxido de metal da superfície com ácido fosfônico ${ }^{80,81,82,83}$ não requer a presença água como reagente, envolve, antes, a condensação não hidrolítica direta de grupos $\mathrm{P}-\mathrm{OH}$ com os grupos hidroxilo da superfície, esquema 3.

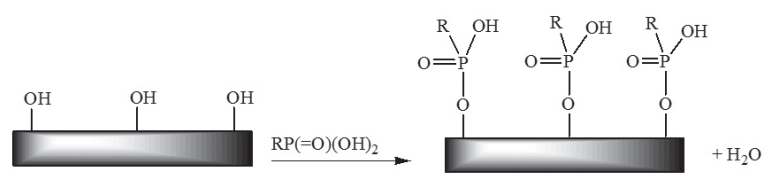

Esquema 3: Reação da superfície hidroxilada com ácido fosfònico.

A modificação da superfície do poro com grupos orgânicos também tem sido realizada com fosfonatos de dietileno em solventes orgânicos a temperaturas moderadas. A clivagem da ligação P-O-C na superfície de óxido de metal, sob condições suaves, sugere que a coordenação dos átomos de oxigênio do fosforilo ao ácido de Lewis, que se encontra à superfície, aumenta a eletrofilicidade do átomo de fósforo, o que facilita a condensação dos grupos P-O-Et com os grupos hidroxilo da superfície, não envolvendo a formação de água, esquema 4.

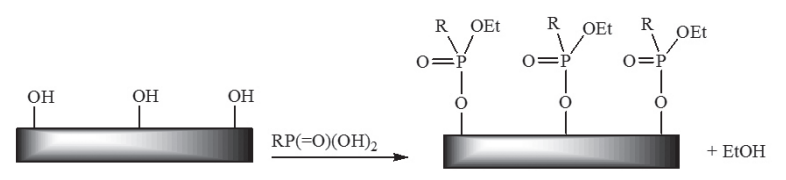

Esquema 4: Reação da superfície hidroxilada com fosfonatos de dietileno.

\section{CONTROLE DA MORFOLOGIA, ESTRUTURA E TEXTURA}

As elevadas energias de ativação envolvidas na rota NHSG refletem-se na redução drástica das taxas de reação e na tendência para favorecer a cristalização controlada e obter formas cristalográficas incomuns. A morfologia do produto final depende fortemente dos parâmetros de síntese que permitem uma versatilidade atrativa para a preparação de produtos bem estruturados. Desse modo, as rotas do éter e alcóxido oferecem uma simples abordagem para a preparação de materiais porosos com elevada área de superfície e elevado volume de poros desordenados na ausência de agentes template e sem secagem supercrítica. Por outro lado, existe também a possibilidade a utilização de templates que fornecem um "escudo" protetor orgânico de forma a dirigir a estrutura/organização desejada.

Dessa forma, o processo não hidrolítico sol-gel oferece múltiplas opções para a síntese de materiais porosos com as propriedades catalíticas, eletrônicas e sinterização pretendidas.

\section{Sem template}

A escolha do solvente é muito importante na transformação sol-gel pois este, em conjunto com os subprodutos da síntese, funcionam como template, uma vez que ficam encurralados no gel formado. Os poros são posteriormente formados por remoção da fase líquida, pelo que a porosidade dos sólidos resultante da rota não hidrolítica depende muito dos parâmetros da síntese como a concentração, o precursor de metal, o doador de oxigênio e o catalisador utilizados. ${ }^{33}$

Para além dos parâmetros de síntese, a porosidade dos materiais depende também do tratamento pós-síntese. Os géis obtidos por via não hidrolítica, que são geralmente mais condensados que os obtidos por vias hidrolíticas, são capazes de resistir às tensões geradas pela evaporação do solvente, mantendo por isso uma elevada porosidade 
após secagem, sem ser necessário o uso de condições supercríticas. O colapso dos poros não ocorre, pois as forças de capilaridade são reduzidas devido às fracas interações entre a superfície (terminada em cloretos e alcóxidos) e a fase líquida. Além deste motivo, o fato de a tensão superficial da fase líquida ser mais baixa do que a da água evita também o colapso dos poros.

Desse modo, foram preparados xerogéis sílica-titânio de mesoporos homogêneos com uma área de superfície entre 450 e $1200 \mathrm{~m}^{2} \mathrm{~g}^{-1}$ e com volume de poros de $0.24 \mathrm{a}$ $2.4 \mathrm{~cm}^{3} \mathrm{~g}^{-1}$ utilizando a via de eliminação de halogeneto de alquilo sem recurso a template ou secagem supercrítica. ${ }^{81}$ A obtenção de xerogéis com esta porosidade mostrou ser controlada pela quantidade de solvente usada e pelo grau de condensação, o que permitiu o ajustamento da textura do material variando apenas o volume de solvente, temperatura ou o tempo de reação.

Também na síntese de nanopartículas, através da reação entre haletos metálicos e o álcool benzílico, é possível controlar o crescimento de cristais, sem a utilização de qualquer ligando adicional e utilizando uma temperatura de $40{ }^{\circ} \mathrm{C} .{ }^{84}$

Os produtos obtidos diretamente do processo de sol-gel não hidrolítico podem ainda ser modificados a posteriori com grupos orgânicos que contribuem para o controlo do tamanho e da forma das estruturas através de ligações de Van der Waals, de interações $\pi-\pi$ e de ligações covalentes. ${ }^{85}$ Esta é uma estratégia importante, pois a imobilização de catalisadores homogêneos sobre uma matriz sólida permite, por um lado, conjugar os elevados desempenhos catalíticos destes (atividade, seletividade, estereosseletividade) com a sua heterogeneização e, por outro, contornar o problema de processamento de espécies ativas que são instáveis ou insolúveis.

\section{Com template}

A maioria das estratégias desenvolvidas para preparar óxidos mesoporosos envolve a adição de agentes orgânicos (templates) que promovem uma porosidade ordenada e estreitas distribuições do tamanho dos poros. O processamento tem por base o self-assembly entre espécies óxido policondensado e o template que é mantido nos produtos sólidos até ao passo da calcinação ou lavagem, em que é eliminado do modelo, como mostra a figura 3 .

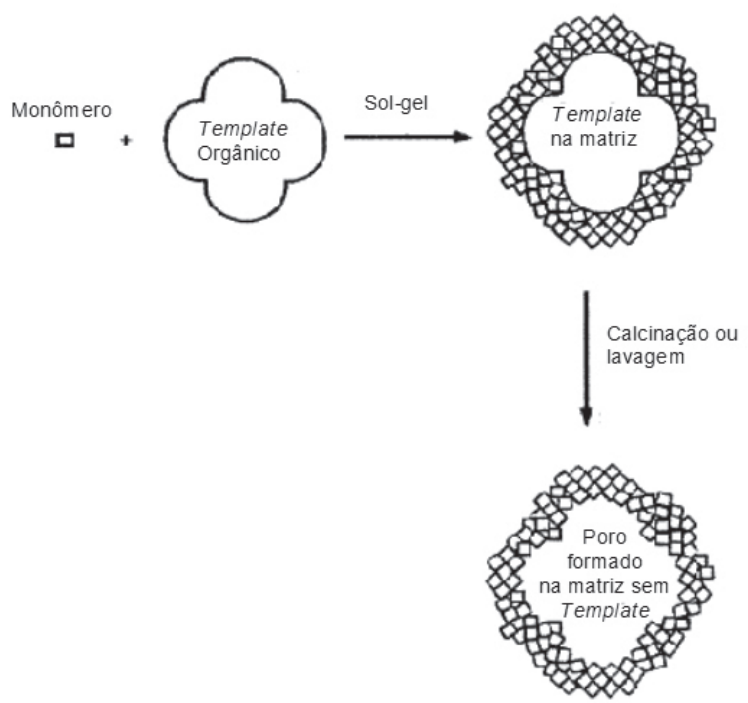

Figura 3: Síntese de materiais inorgânicos recorrendo ao template

A formação de micelas ou mesofases é feita com base em interações hidrofóbicas/hidrofílicas e, portanto a maioria dos procedimentos envolvem o uso de água. Porém, Stucky et al. ${ }^{86,87}$ prepararam óxidos de metais com mesoporos ordenados baseados na reação de cloretos metálicos com álcoois na presença de um template anfifílico. Reações não hidrolíticas estiveram, provavelmente, envolvidas na síntese destes materiais mesoporosos, embora a água (resultante da umidade atmosférica ou partir do solvente ou precursores) estivesse presente no processo.

Tilley e os seus colaboradores utilizaram a decomposição termolítica de derivados de tercbutoxi(siloxanos) na presença de blocos de copolímeros para obter óxidos mesoporosos, constituídos por vários metais, com elevada área de superfície e com poros com estreitas distribuições e com estrutura do tipo wormhole. ${ }^{88}$

Embora o uso de templates tenha levado à síntese de materiais com poros que apresentam áreas de superfície e volume que variam entre $450-550 \mathrm{~m}^{2} \mathrm{~g}^{-1} \mathrm{e}$ $0.35-0.45 \mathrm{~cm}^{3} \mathrm{~g}^{-1}$, respetivamente, o processamento sem template permite também obter materiais com os poros com uma área superficial muito próxima $\left(415-555 \mathrm{~m}^{2} \mathrm{~g}^{-1}\right)$ e volumes maiores $\left(0.7-1.0 \mathrm{~cm}^{3} \mathrm{~g}^{-1}\right)$. 
A rota não hidrolítica tem sido também empregue na síntese de nanopartículas mesoestruturadas na presença de blocos de copolímeros. Nanopartículas de óxido de estanho e óxido de cério foram obtidas através da rota eliminação do álcool e dispersas em THF, sem a utilização de estabilizadores. A adição de um bloco copolímero (polibutadieno-poli(óxido etileno) e evaporação do THF, induz o self-assembly dos nanocristais com micelas do copolímero. ${ }^{89}$ Após calcinação, foram obtidos nanopartículas mesoporosas de óxido de estanho e cério altamente ordenadas.

Outra estratégia mais recente, ${ }^{90}$ baseia-se na utilização de bloco de copolímero de poli-isopreno insaturado, que pode ser convertido numa fase amorfa de carbono mantendo os poros intatos durante a cristalização, sob atmosfera inerte. Após remoção do carbono amorfo por calcinação a $450^{\circ} \mathrm{C}$, foram obtidos óxidos metálicos cristalinos com mesoporos bem ordenados e com uma área superficial entre 54 e $89 \mathrm{~m}^{2} \mathrm{~g}^{-1}$.

\section{Aplicação de materiais porosos em catálise heterogênea}

Os óxidos metálicos representam uma das mais importantes e amplas categorias de catalisadores na área da catálise heterogênea, quer como fases ativas quer como suportes. A utilização de metais de transição e metais nobres em materiais porosos tem permitido a sua aplicação como catalisadores. Dentre os catalisadores de óxido de metal, os de metais de transição ocupam um lugar de destaque, devido ao seu baixo custo de produção, fácil reutilização e elevada seletividade. Por este motivo, estes catalisadores têm vindo a ser aplicados em muitas reações orgânicas, síntese em química fina, química verde e há já aplicações industriais na área do petróleo, figura 4.

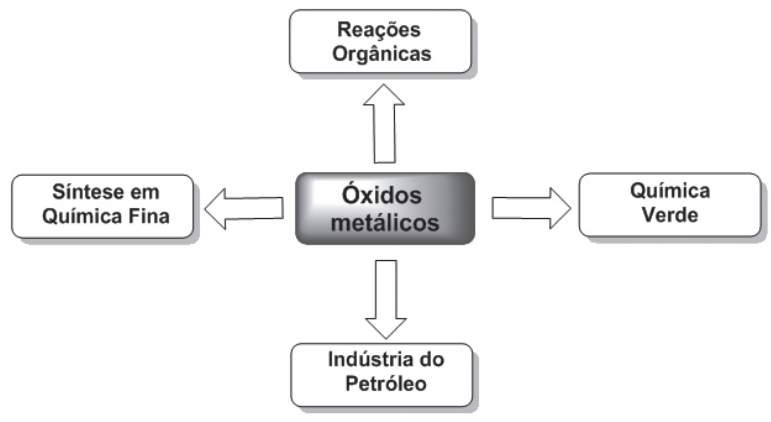

Figura 4: Aplicações catalíticas dos óxidos metálicos.
Os materiais porosos preparados pela rota NHSG, como já foi discutido nos tópicos anteriores, possuem áreas de superfície maiores, o que reduz o tempo de reação e aumenta o rendimento ou conversões das reações. Estas caraterísticas, associadas à simplicidade de manuseio, diminuição do tamanho do reator, diminuição de problemas de corrosão, rentabilidade e possibilidade de reclicagem e reutilização, fazem desta abordagem uma boa estratégia futura, em especialmente em nível industrial.

Na seção seguinte será abordada a utilização de óxidos de metais, dentro das áreas representadas na figura 4 , na promoção de alguns tipos de reações.

\section{REAÇÕES DE OXIDAÇÃO}

Os óxidos metálicos são muito usados como catalisadores em oxidações seletivas, tendo, por isso, grande impato na indústria.

Catalisadores heterogêneos à base de óxidos de $\mathrm{Nb}-\mathrm{V}$, $\mathrm{Nb}$-V-Si e Cr-Si têm sido utilizados em desidrogenações oxidativa de alcanos. As formulações catalíticas, baseadas em vanádio e nióbio, estão entre as mais estudadas, pois o óxido de nióbio é pouco ativo, mas muito seletivo enquanto que o óxido de vanádio é bastante ativo sob condições oxidações, mas pouco seletivo. A sua grande utilização é também devido ao fato de estes serem sintetizados, pela rota não hidrolítica da eliminação do haleto de alquilo, com elevado grau de homogeneidade o que permite controlar a sua atividade catalítica. ${ }^{93,74,94,95}$

Por outro lado, materiais inorgânicos de silício (óxidos Ti-Si e Ta-Si) têm sido empregues em reações de oxidativas e epoxidações, com peróxido de hidrogênio, sob condições moderadas. ${ }^{96}$ Este tipo de catalisadores era de difícil obtenção através de rotas sol-gel convencionais ou eram apenas utilizados na catálise de substratos pequenos devido ao pequeno tamanho dos poros. Contudo, a utilização de rotas não hidrolíticas melhorou significativamente o volume e área de poros dos materiais obtidos o que tem permitido a sua aplicação em substratos mais volumosos. ${ }^{67,81}$ Além disto, a atividade e seletividade de catalisadores de $\mathrm{SiO} 2-\mathrm{TiO} 2$ pode ser ajustada através de modificações durante ou pós-síntese, como é exemplo a introdução de grupos hidrofóbicos orgânicos ( $\mathrm{SiMe}, \mathrm{SiPh}$ ou SiMe3). ${ }^{97,98,99}$

Ciuffi e os seus colaboradores utilizaram a via do éter para preparar óxidos de Co-Al-Si e Co-Al, que 
aplicaram na oxidação de hidrocarbonetos. Verificaram que não ocorria lixiviação do cobalto, ao contrário do que acontecia com as amostras preparadas pela via hidrolítica de sol-gel. ${ }^{100,78}$ Esta via foi também utilizada para preparar óxidos de $\mathrm{Fe}-\mathrm{Al}$ que foram aplicados na oxidação seletiva de ciclo-hexeno e ciclo-hexano. ${ }^{101}$

Óxidos mistos de vanádio-titânio (V-Ti, V-Mo-Ti, V-W-Ti), por sua vez, têm vindo a ser muito estudados e explorados industrialmente na oxidação total de diversos poluentes orgânicos atmosféricos..$^{102,103} \mathrm{~A}$ rota não hidrolítica veio colmatar algumas limitações das rotas convencionais onde, para a obtenção deste tipo de materiais, eram necessários vários passos reacionais. Adicionalmente, na calcinação, ocorre a migração de espécies de vanádio para a superfície dos materiais, o que aumenta a atividade desses.

Além destes exemplos, também o catalisador de Jacobsen, encapsulado em matrizes de alumina, figura 3, tem sido utilizado na oxidação seletiva de ciclohexano, estireno e ciclo-octeno com recurso a diferentes oxidantes. ${ }^{85}$

\section{REAÇÕES DE REDUÇÃO}

A redução de poluentes orgânicos, entre os quais NOx, é cada vez mais importante devido à forte vigilância das entidades reguladoras desta área. As regulamentações são cada vez mais rigorosas e existe uma forte preocupação com o ambiente, principalmente, com a libertação, para o ar, de NOx. É neste contexto que os materiais porosos inorgânicos à base de óxidos de metais se podem apresentar como uma importante ferramenta nesta área, uma vez que podem catalisar a redução destes poluentes.

Para este efeito, óxidos mistos de V-Ti têm sido utilizados na redução catalítica seletiva de NOx recorrendo a amoníaco como agente redutor. Elaborados procedimentos foram propostos para aumentar a atividade destes catalisadores de V-Ti, mas o processo sol-gel não hidrolítico usando precursores modificados quimicamente, procedimentos para promover a dispersão das espécies de vanádio ou a utilização da secagem supercrítica permitiram aumentar a área de superficie do material. ${ }^{76,77}$ A utilização deste catalisador, neste tipo de reações, requer a presença de agentes redutores o que é uma desvantagens na aplicação, por exemplo, em veículos. Por esta razão, os catalisadores suportados em óxidos mistos de prata-alumínio (Ag-Al e Ag-Nb-Al) são muito atraentes para a redução de $\mathrm{NOx}$ dado que utilizam hidrocarbonetos, também produzidos na combustão dos motores, como agentes redutores. Para este efeito, apesar de os óxidos mistos de Ag-Al serem os mais ativos e seletivos, os catalisadores utilizados possuem também nóbio, pois a sua presença aumenta bastante a área de superfície e o tamanho dos poros, o que conduz a um aumento significativo da atividade à baixa temperatura. ${ }^{104,105}$ Todas estas modificações que conduziram a melhorias na atividade e seletividade dos catalisadores são resultado da utilização de rotas não hidrolíticas de sol-gel.

\section{REAÇÕES DE ALQUILAÇÃO}

Nanopartículas de óxido de estanho preparadas através do aquecimento de $\mathrm{SnCl} 4$ em álcool benzílico têm sido utilizadas para promover reações de alquilação. No mecanismo proposto, o átomo de cloro à superfície da nanopartícula pode ser transferido para álcool benzílico, originando cloreto de benzilo. Este atua como agente de alquilação e no final o cloro retorna à superfície da nanopartícula. ${ }^{106}$

Este tipo de nanopartículas também tem sido utilizado para catalisar a alquilação de Friedel-Crafts de compostos aromáticos (benzeno, tolueno e metoxibenzeno) com vários álcoois benzílicos.

A síntese de catalisadores capazes de promover estas reações só é possível através da rota NHSG, pois a presença de átomos de cloro na superfície das nanopartículas é mesmo necessária, sendo que de outro modo os catalisadores são inativos.

\section{REAÇÕES DE POLIMERIZAÇÃO/ OLIGOMERIZAÇÃO}

Os metalocenos são largamente usados como catalisadores para a polimerização de olefinas, devido à sua elevada atividade e estereoseletividade. Por este motivo, muito tempo se dedicou à sua heterogeneização, especialmente em matrizes de sílica ${ }^{107,108} \mathrm{Si}^{-\mathrm{Ti}^{109}}$ e Si-W ${ }^{110}$ preparadas por NHSG. O catalisador, cuja imobilização consistiu no encapsulamento nas matrizes sólidas, apresentou uma atividade moderada para a polimerização de olefinas, não se verificando a ocorrência de lixiviação.

A oligomerização de álcoois benzílicos utilizando nanofios de $\mathrm{W}_{18} \mathrm{O}_{49}$, formados a através do aquecimento 
de isopropóxido de tungstênio em álcool benzílico foi também reportada, neste contexto, por Olliges-Stadler e colaboradores. ${ }^{111}$

\section{REAÇÕES DE HIDROGENAÇÃO}

Para esta reação, Anderson e os seus colaboradores descreveram a encapsulação de nanopartículas de paládio numa matriz de sílica, preparada por vias não hidrolíticas utilizando líquidos iônicos como solventes. Este material foi avaliado na hidrogenação do cinamaldeído, em tolueno, a $80^{\circ} \mathrm{C}$ e a uma pressão de 10 bar de hidrogênio, tendo sido observada conversão completa após $1 \mathrm{~h} .{ }^{112}$

A mesma técnica foi utilizada para o encapsulamento de $\left[\mathrm{RhCl}\left(\mathrm{PPH}_{3}\right)_{3}\right]$ e $\left.\left[\mathrm{Rh}_{2}(\mathrm{COD})_{2}(\mathrm{dppm})\left(\mu_{2}-\mathrm{Cl}\right)\right] \mathrm{BF}_{4}\right] \mathrm{em}$ matrizes de sílica. A área de superfície específica do material obtido foi relativamente baixa $\left(<20 \mathrm{~m}^{2} \mathrm{~g}_{-}{ }^{1}\right)$, o que foi atribuído ao aprisionamento de líquidos iônicos. Para comparação, foi também realizado o encapsulamento de $\left[\mathrm{RhCl}\left(\mathrm{PPH}_{3}\right)_{3}\right]$ e $\left[\mathrm{Rh}_{2}(\mathrm{COD})_{2}(\mathrm{dppm})\left(\mu_{2}-\mathrm{Cl}\right)\right]$ $\mathrm{BF}_{4}$ ] em matrizes de sílica por processos hidrolíticos convencionais (na ausência de líquidos iônicos). Todos os materiais foram testados como catalisadores na hidrogenação do estireno a $100{ }^{\circ} \mathrm{C}$ e 25 bar de pressão de $\mathrm{H}_{2}$ e a sua atividade foi comparada com o respetivo catalisador homogêneo. $\mathrm{O}\left[\mathrm{RhCl}\left(\mathrm{PPH}_{3}\right)_{3}\right]$ encapsulado, por ambas as vias descritas anteriormente, foi bastante mais ativo quando comparado com o catalisador homogéneo e praticamente não se verificou lixiviação.

\section{FOTOCATÁLISE}

A via não hidrolítica sol-gel tem um papel importante na degradação fotocatalítica heterogênea de poluentes orgânicos em águas residuais. O dióxido de titânio $\left(\mathrm{TiO}_{2}\right)$ é um dos mais utilizados devido à sua abundância, baixo custo, facilidade de processamento e excelente atividade catalítica. Contudo, o titânio apresenta limitações para esta aplicação como uma band gap larga e uma alta taxa de recombinação de eletrões fotogerados. Neste sentido, a preparação de materiais de $\mathrm{TiO}_{2}$ modificados de forma a reduzir a band gap e aumentar a separação de carga de eletrões fotogerados é necessária para aumentar a atividade fotocatalítica dos materiais obtidos. As estratégias utilizadas neste sentido centram-se na dopagem do $\mathrm{TiO}_{2}$ com átomos de $\mathrm{N}, \mathrm{P}$ ou metais de transição ou na dispersão de espécies de $\mathrm{TiO}_{2}$ em zeólitos ou matrizes de sílica. ${ }^{115,116,117}$ Assim, a rota não hidrolítica assume um papel importante, pois está bem estabelecido que as propriedades catalíticas destes materiais dependem da morfologia, fase cristalina, área superficial, tamanho de partícula e estrutura eletrônica.

\section{METÁTESE DE ALCENOS}

A metátese de olefinas, com base em catalisadores de $\mathrm{MoO}_{3}$, é uma reação de grande importância na síntese orgânica, na química da polimerização e na indústria (petroquímica). Os catalisadores são, principalmente, preparados através da impregnação de Mo em suportes de sílica, alumina, titânio e sílica-alumínio. ${ }^{118,119}$ Foi observado, com base em trabalhos experimentais e estudos teóricos, que para que o catalisador seja ativo é necessário que o suporte tenha acidez e que as espécies de MoOx estejam altamente dispersas.

Neste contexto, materiais à base de óxidos mistos de Mo-Al-Si foram preparados por via não hidrolítica de sol-gel e avaliados na metátese de propeno para eteno e buteno, exibindo atividade mais elevada do que outros sistemas de referência. ${ }^{120}$

\section{Conclusão}

O processo não hidrolítico de sol-gel complementa o processo sol-gel convencional, oferecendo vias versáteis que já provaram ser particularmente úteis para a preparação de uma grande variedade de catalisadores heterogêneos.

A principal vantagem da utilização de processos não hidrolíticos é possibilidade de controle da estrutura e composição, em nível molecular, dos óxidos de metal. Muitos exemplos aqui apresentados mostram que a rota não hidrolítica veio solucionar muitos problemas do processo de sol-gel convencional, pois permitiu a síntese, num só passo, de catalisadores mistos com controlada homogeneidade composicional e excelentes propriedades texturais, partindo de precursores de cloreto de baixo custo e evitando o uso de modificadores de reatividade.

A alteração das reações que levam à formação das ponte oxo e a alteração do meio reacional, leva a que a cinética de reação, o grau de condensação e a natureza dos grupos residuais no gel sejam alterados. Todos estes aspetos fazem com que a homogeneidade, textura e propriedades de superfície dos materiais obtidos sejam afetadas. 
A versatilidade deste método permite também a fácil incorporação de dopantes ou grupos orgânicos, durante o processo de síntese do material ou a posteriori, e as condições não aquosas utilizadas por esta rota facilitam a imobilização de espécies catalíticas sensíveis ou pouco solúveis em água como, por exemplo, complexos organometálicos, proporcionando oportunidades únicas para a descoberta de novos catalisadores com maior desempenhos catalíticos.

Há ainda muito a ser feito para explorar todas as possibilidades oferecidas por este processo, que terá crescente aplicação no design de novos materiais.

\section{Referências}

1. B. L. Cushing, V. L. Kolesnichenko, C. J. O Connor, Chem. Rev., 2004, 104, 3893.

2. L. B. Capeletti, "Efeitos da rota sol-gel no encapsulamento de indicadores colorimétricos e fluorimétricos e em suas performances como sensores de $\mathrm{pH}$ e gás amônia”, Porto Alegre, 2012.

3. B. E. Yoldas, J. Mater. Sci., 1986, 21, 1086.

4. C. Sanchez, J. Livage, M. Henry, F. Babonneau, J. Non-Cryst. Solids, 1988, 100, 65.

5. G. M. Pajonk, Appl. Catal., 1991, 72, 217.

6. S. S. Prakash, C. J. Brinker, A. J. Hurd, S. M. Rao, Nature, 1995, 374, 439 .

7. A. Taguchi, F. Schuth, Microporous Mesoporous Mater., 2005, $77,1$.

8. A. Corma, Chem. Rev., 1997, 97, 2373.

9. D. P. Debecker, M. Stoyanova, F. C.-Justin, U. Rodemerck, C. Boissiere, E. M. Gaigneaux, C. Sanchez, Angew. Chem., Int. Ed., 2012, 51, 2129.

10. R. Corriu, D. Leclercq, P. Lefevre, P. H. Mutin, A. Vioux, Chem. Mater., 1992, 4, 961.

11. R. J. P. Corriu, D. Leclercq, P. Lefevre, P. H. Mutin, A. Vioux, J. Mater. Chem., 1992, 2, 673.

12. R. J. P. Corriu, D. Leclercq, P. Lefevre, P. H. Mutin, A. Vioux, J. Non-Cryst. Solids, 1992, 146, 301.

13. A. Vioux, Chem. Mater., 1997, 9, 2292.

14. P. H. Mutin, A. Vioux, Chem. Mater., 2009, 21, 582.

15. J. N. Hay, H. M. Raval, Chem. Mater., 2001, 13, 3396.

16. N. Pinna, J. Mater. Chem., 2007, 17, 2769.

17. Y.-w. Jun, J.-s. Choi, J. Cheon, Angew. Chem., Int. Ed., 2006, 45, 3414.
18. M. Niederberger, Acc. Chem. Res., 2007, 40, 793.

19. M. Leskela, M. Ritala, Angew. Chem., Int. Ed., 2003, 42, 5548.

20. G. Clavel, E. Rauwel, M. G. Willinger, N. Pinna, J. Mater. Chem., 2009, 19, 454.

21. N. Pinna, M. Niederberger, Angew. Chem., Int. Ed., 2008, 47, 5292.

22. G. Garnweitner, M. Niederberger, J. Mater. Chem., 2008, 18, 1171.

23. L. Bourget, R. J. P. Corriu, D. Leclercq, P. H. Mutin, A. Vioux, J. Non-Cryst. Solids, 1998, 242, 81.

24. M. Andrianainarivelo, R. Corriu, D. Leclercq, P. H. Mutin, A. Vioux, J. Mater. Chem., 1996, 6, 1665.

25. M. Andrianainarivelo, R. J. P. Corriu, D. Leclercq, P. H. Mutin, A. Vioux, J. Mater. Chem., 1997, 7, 279.

26. M. Andrianainarivelo, R. J. P. Corriu, D. Leclercq, P. H. Mutin, A. Vioux, Chem. Mater., 1997, 9, 1098.

27. A. P. Wilkinson, C. Lind, S. Pattanaik, Chem. Mater., 1999, 11, 101.

28. S. D. Gates, J. A. Colin, C. Lind, J. Mater. Chem., 2006, 16, 4214.

29. P. H. Mutin, A. Vioux, Chem. Mater., 2009, 21, 596.

30. V. G. Kessler, Chem. Commun. 20031213.

31. V. G. Kessler, K. V. Nikitin, A. I. Belokon, Polyhedron, 1998, 17, 2309.

32. E. Stathatos, P. Lianos, U. Lavrencic-Stangar, B. Orel, AdV. Mater., 2002, 14, 354.

33. C. Wang, Z. Deng, Y.-D. Li, Inorg. Chem., 2001, 40, 5210.

34. J. Caruso, M. J. Hampden-Smith, J. Sol-Gel Sci. Technol., 1997, 8,35 .

35. M. Jansen, E. Guenther, Chem. Mater., 1995, 7, 2110.

36. K. G. Sharp, AdV. Mater., 1998, 10, 1243.

37. S. Dai, Y. H. Ju, H. J. Gao, J. S. Lin, S. J. Pennycook, C. E. Barnes, Chem. Commun., 2000, 3, 243.

38. M.-A. Neouze, J. Le Bideau,F. Leroux, A. Vioux, Chem. Commun., 20051082.

39. R. Sui, A. S. Rizkalla, P. A. Charpentier, J. Phys. Chem. B, 2004, 108,11886

40. P. Arnal, R. J. P. Corriu, D. Leclercq, P. H. Mutin, A. Vioux, J. Mater. Chem., 1996, 6, 1925.

41. M. Fujiwara, H. Wessel,H. S. Park, H. W. Roesky, Chem. Mater., 2002, 14, 4975.

42. Z. Zhang, X. Zhong, S. Liu, D. Li, M. Han, Angew. Chem., Int. Ed. $2005,44,3466$.

43. P. D. Cozzoli, M. L. Curri, A. Agostiano,G. Leo, M. Lomascolo, J. Phys. Chem. B, 2003, 107, 4756. 
44. N. Steunou, F. Ribot, K. Boubekeur, J. Maquet, C. Sanchez, New J. Chem., 1999, 23, 1079.

45. G. Garnweitner, M. Antonietti, M. Niederberger, Chem. Commun., 2005397.

46. S. Zhou, M. Antonietti, M. Niederberger, Small, 2007, 3, 763.

47. M. Guerbet, Compt. Rend., 1902, 135, 172.

48. M. Niederberger, G. Garnweitner, N. Pinna, M. Antonietti, J. Am. Chem. Soc., 2004, 126, 9120.

49. B. Koo, J. Park, Y. Kim, S.-H. Choi, Y.-E. Sung, T. Hyeon, J. Phys. Chem. B, 2006, 110, 24318.

50. Y. W. Jun, M. F. Casula, J.-H. Sim, S. Y. Kim, J. Cheon, A. P. Alivisatos, J. Am. Chem. Soc., 2003, 125, 15981.

51. J. Joo, S. G. Kwon, J. H. Yu, T. Hyeon, Adv. Mater., 2005, 17, 1873.

52. K. W. Terry, T. D. Tilley, Chem. Mater., 1991, 3, 1001.

53. K. L. Fujdala, T. D. Tilley, Chem. Mater., 2001, 13, 1817.

54. J. Jarupatrakorn, T. D. Tilley, J. Am. Chem. Soc., 2002, 124, 8380.

55. C. Nozaki, C. G. Lugmair, A. T. Bell, T. D. Tilley, J. Am. Chem. Soc., 2002 , 124, 13194.

56. J. N. Hay, H. M. Raval, J. Mater. Chem., 1998 , 8, 1233.

57. V. Lafond, P. H. Mutin, A. Vioux, A. Chem. Mater., 2004, 16, 5380 .

58. S. Acosta, R. Corriu, D. Leclercq, P. H. Mutin, A. Vioux, J. SolGelSci. Technol., 1994, 2, 25.

59. D. Janackovic, A. Orlovic, D. Skala, S. Drmanic, L. K. KosticGvozdenovic, V. Jokanovic, D. Uskokovic, Nanostruct. Mater., 1999, 12, 147.

60. J. H. Harreld, J. Sakamoto, B. Dunn, J. Power Sources, 2003, 115, 19.

61. R. Linacero, M. L. Rojas-Cervantes, J. D. D. Lopez-Gonzalez, J. Mater. Sci., 2000, 35, 3269.

62. M. Mann, G. E. Shter, G. S. Grader, J. Mater. Res., 2002, 17, 831.

63. J. Xu, C. Lind,A. P. Wilkinson, S. Pattanaik, Chem. Mater., 2000, $12,3347$.

64. F. Barbieri, D. Cauzzi, F. De Smet, M. Devillers, P. Moggi, G. Predieri, P. Ruiz, Catal. Today, 2000, 61, 353.

65. W. Huang, X. Tang, I. Felner, Y. Koltypin, A. Gedanken, Mater. Res.Bull., 2002, 37, 1721.

66. A. F. Popa, P. H. Mutin, A. Vioux, G. Delahay, B. Coq, Chem. Commun., 20042214.

67. P. H. Mutin, A. F. Popa, A. Vioux, G. Delahay, B. Coq, Appl. Catal., B, 2006, 69, 49.

68. B. L. Caetano, L. A. Rocha, E. Molina, Z. N. Rocha, G. Ricci, P. S. Calefi, O. J. de Lima, C. Mello, E. J. Nassar, K. J. Ciuffi, Appl. Catal., A, 2006, 311, 122.
69. M. L. Di Vona, E. Traversa, S. Licoccia, Chem. Mater., 2001, 13, 141.

70. O. Lorret, V. Lafond, P. H. Mutin, A. Vioux, Chem. Mater., 2006, $18,4707$.

71. A. M. Cojocariu, P. H. Mutin, E. Dumitriu, F. Fajula, A. Vioux, V. Hulea, Chem. Commun. 20085357.

72. V. Lafond, P. H. Mutin, A. Vioux, J. Mol. Catal. A, 2002, 81, 182.

73. P. Ratnasamy, D. Srinivas, H. Knözinger, Adv. Catal., 2004, 48, 1.

74. O. J. de Lima, D. P. de Aguirre, D. C. de Oliveira, M. A. da Silva, C. Mello, C. A. P. Leite, H. C. Sacco, K. J. Ciuffi, J. Mater. Chem., 2001, 11, 2476.

75. T. C. O. Mac Leod, D. F. C. Guedes, M. R. Lelo, R. A. Rocha, B. L. Caetano, K. J. Ciuffi, M. D. Assis, J. Mol. Catal. A, 2006, 259, 319.

76. J. M. A. Caiut, S. Nakagaki, O. J. De Lima, C. Mello, C. A. P. Leite, E. J. Nassar, K. J. Ciuffi, H. C. Sacco, J. Sol-Gel Sci. Technol., 2003, 28, 57.

77. A. Ulman, Chem. Rev., 1996, 96, 1533.

78. C. P. Tripp, M. L. Hair, Langmuir, 1995, 11, 1215.

79. A. Y. Fadeev, T. J. McCarthy, J. Am. Chem. Soc., 1999, 121, 12184

80. W. Gao, L. Dickinson, C. Grozinger, F. G. Morin, L. Reven, Langmuir, 1996, 12, 6429.

81. R. Helmy, A. Y. Fadeev, Langmuir, 2002, 18, 8924.

82. E. S. Gawalt, M. J. Avaltroni, M. P. Danahy, B. M. Silverman, E. L. Hanson, K. S. Midwood, J. E. Schwarzbauer, J. Schwartz, Langmuir, 2003, 19, 200.

83. P. H. Mutin, G. Guerrero, A. Vioux, J. Mater. Chem., 2005, 15, 3761

84. M. Niederberger, M. H. Bartl, G. D. Stucky, Chem. Mater., 2002, 14,4364 .

85. M. Antonietti, M. Niederberger, B. Smarsly, Dalton Trans., 2008 18.

86. P. D. Yang, D. Y. Zhao, D. I. Margolese, B. F. Chmelka, G. D. Stucky, Nature, 1998, 396, 152.

87. P. D. Yang, D. Y. Zhao, D. I. Margolese, B. F. Chmelka, G. D. Stucky, Chem. Mater., 1999, 11, 2813.

88. J. W. Kriesel, M. S. Sander, T. D. Tilley, Adv. Mater., 2001, 13, 331

89. J. Ba, J. Polleux, M. Antonietti, M. Niederberger, Adv. Mater., 2005, 17,2509

90. A. S. Deshpande, N. Pinna, B. Smarsly, M. Antonietti, M. Niederberger, Small, 2005, 1, 313.

91. A. P. Amrute, A. Bordoloi, N. Lucas, K. Palraj, S. B. Halligudi, Catal. Lett., 2008, 126, 286. 
92. S. J. Singh, R. V. Jayaram, Tetrahedron Lett., 2008, 49, 4249.

93. T. C. Watling, G. Deo, K. Seshan, I. E. Wachs, J. A. Lercher, Catal. Today, 1996, 28, 139.

94. P. Moggi, G. Predieri, D. Cauzzi, M. Devillers, P. Ruiz, S. Morselli, O. Ligabue, Stud. Surf. Sci. Catal., 2002, 143, 149.

95. P. Moggi, M. Devillers, P. Ruiz, G. Predieri, D. Cauzzi, S. Morselli, O. Ligabue, Catal. Today, 2003, 81, 77.

96. R. A. Sheldon, M. Wallau, I. W. C. E. Arends, U. Schuchardt, Acc. Chem. Res., 1998, 31, 485.

97. R. Mariscal, M. Lopez-Granados, J. L. G. Fierro, J. L. Sotelo, C. Martos, R. Van Grieken, Langmuir, 2000, 16, 9460.

98. T. Tatsumi, K. A. Koyano, N. Igarashi, Chem. Commun., 1998 325.

99. Y. Deng, W. F. Maier, J. Catal., 2001, 199, 115.

100.O. J. de Lima, A. T. Papacidero, L. A. Rocha, H. C. Sacco, E. J. Nassar, K. J. Ciuffi, L. A. Bueno, Y. Messaddeq, S. J. L. Ribeiro, Mater. Charact., 2003, 50, 101.

101.G. P. Ricci, Z. N. Rocha, S. Nakagaki, K. A. D. F. Castro, A. E. M. Crotti, P. S. Calefi, E. J. Nassar, K. J. Ciuffi, Appl. Catal., A, 2010, 389, 147.

102.R. Delaigle, D. P. Debecker, F. Bertinchamps, E. M. Gaigneaux, Top. Catal., 2009, 52, 501.

103.W. B. Li, J. X. Wang, H. Gong, Catal. Today, 2009, 148, 81.

104.P. Forzatti, L. Lietti, Heterog. Chem. Rev., 1996, 3, 33.

105.D. P. Debecker, P.H. Mutin, Chem. Soc. Rev., 2012, 41, 3624.

106.P. Makowski, R. Rothe, A. Thomas, M. Niederberger, F. Goettmann, Green Chem., 2009, 11, 34.

107.A. Fisch, C. F. Petry, D. Pozebon, F. C. Stedile, N. S. M. Cardozo, A. R. Secchi, J. H. Z. dos Santos, Macromol. Symp., 2006, 245246, 77.

108.A. G. Fisch, N. S. M. Cardozo, A. R. Secchi, F. C. Stedile, N. P. da Silveira, J. H. Z. dos Santos, J. Non-Cryst. Solids, 2008, 354, 3973.

109.A. G. Fisch, N. S. M. Cardozo, A. R. Secchi, F. C. Stedile, P. R. Livotto, D. S. de Sa, Z. N. da Rocha and J. H. Z. Dos Santos, Appl. Catal., A, 2009, 354, 88.

110.A. G. Fisch, N. S. M. Cardozo, A. R. Secchi, F. C. Stedile, C. Radtke, D. S. De Sa, Z. N. da Rocha, J. H. Z. dos Santos, Appl. Catal., A, 2009 , 370, 114.

111. I. Olliges-Stadler, M. D. Rossell, M. Niederberger, Small, 2010, 6,960 .

112.K. Anderson, S. Cortinas Fernandez, C. Hardacre, P. C. Marr, Inorg. Chem. Commun., 200473.

113. S. J. Craythorne, A. R. Crozier, F. Lorenzini, A. C. Marr, P. C. Marr, J. Organomet. Chem., 2005, 690, 3518.

114. S. J. Craythorne, K. Anderson, F. Lorenzini, C. McCausland, E.
F. Smith, P. Licence, A. C. Marr, P. C. Marr, Chem.-Eur. J., 2009, 15,7094 .

115.R. Asahi, T. Morikawa, T. Ohwaki, K. Aoki, Y. Taga, Science, 2001, 293, 269.

116.A. M. Tripathi, R. G. Nair, S. K. Samdarshi, Sol. Energy Mater. Sol. Cells, 2010, 94, 2379.

117.X. Chen, S. S. Mao, Chem. Rev., 2007, 107, 2891.

118.D. P. Debecker, D. Hauwaert, M. Stoyanova, A. Barkschat, U. Rodemerck, E. M. Gaigneaux, Appl. Catal., A, 2011, 391, 78.

119.J. Handzlik, J. Ogonowski, J. Stoch, M. Mikolajczyk, Appl. Catal., A, 2004, 273, 99.

120.D. P. Debecker, K. Bouchmella, C. Poleunis, P. Eloy, P. Bertrand, E. M. Gaigneaux, P. H. Mutin, Chem. Mater., 2009, 21, 2817.

\section{César A. S. Henriques ${ }^{1 *}$, Lucas D. Dias', Mário J. F. Calvete' \& Gilberto L. B. de Aquino $^{2}$}

${ }^{1}$ CQC, Departamento de Química, Universidade de Coimbra, Coimbra, Portugal,

${ }^{2}$ Unidade de Ciências Exatas e Tecnológicas, Universidade Estadual de Goiás, Anápolis, Goiás, Brasil.

*Email: c.henriques@qui.uc.pt 